\title{
Obstructions to chordal circular-arc graphs of small independence number
}

\author{
Mathew Francis ${ }^{\mathrm{a}, 1}$, Pavol Hell ${ }^{\mathrm{b}, 2}$, Juraj Stacho ${ }^{\mathrm{c}, 3, *}$ \\ ${ }^{a}$ Institute of Mathematical Sciences, IV Cross Road, CIT Campus, Taramani, Chennai 600 113, India \\ ${ }^{b}$ School of Computing Science, Simon Fraser University, 8888 University Drive, Burnaby, Canada V5A 1 S6 \\ ${ }^{c}$ DIMAP and Mathematics Institute, University of Warwick, Coventry CV4 7AL, United Kingdom
}

\begin{abstract}
A blocking quadruple (BQ) is a quadruple of vertices of a graph such that any two vertices of the quadruple either miss (have no neighbours on) some path connecting the remaining two vertices of the quadruple, or are connected by some path missed by the remaining two vertices. This is akin to the notion of asteroidal triple used in the classical characterization of interval graphs by Lekkerkerker and Boland [11].

We show that a circular-arc graph cannot have a blocking quadruple. We also observe that the absence of blocking quadruples is not in general sufficient to guarantee that a graph is a circular-arc graph. Nonetheless, it can be shown to be sufficient for some special classes of graphs, such as those investigated in [2].

In this note, we focus on chordal graphs, and study the relationship between the structure of chordal graphs and the presence/absence of blocking quadruples.

Our contribution is two-fold. Firstly, we provide a forbidden induced subgraph characterization of chordal graphs without blocking quadruples. In particular, we observe that all the forbidden subgraphs are variants of the subgraphs forbidden for interval graphs [11]. Secondly, we show that the absence of blocking quadruples is sufficient to guarantee that a chordal graph with no independent set of size five is a circulararc graph. In our proof we use a novel geometric approach, constructing a circular-arc representation by traversing around a carefully chosen clique tree.
\end{abstract}

Keywords: circular-arc graph, chordal graph, forbidden subgraph characterization, asteroidal triple

\section{Introduction}

The study of graph obstructions has a long tradition in graph theory. To understand the structure of graphs in a particular graph class, it is often useful (if not easier) instead to characterize all minimal graphs that are not in the class, usually known as obstructions. They often result in elegant characterization theorems and can be used as succinct certificates in certifying algorithms.

In this paper, we seek obstructions to circular-arc graphs, the intersection graphs of families of arcs of a circle. This problem dates back at least as far as the 1970's $[9,13,14,15,16]$, and remains a challenging question capturing the interest of many researchers over the years $[1,2,6,9,12,13,16,14,15]$.

Predating the study of circular arc graphs, the class of interval graphs, intersection graphs of families of intervals of the real line, was investigated. Interval graphs are a subclass of chordal graphs, graphs in which every cycle has a chord, as well as of circular-arc graphs. They are known to admit a number of interesting characterizations $[8,11]$ and efficient recognition algorithms $[3,5,10]$. In particular, the result of Lekkerkerker and Boland [11] describes interval graphs in terms of forbidden induced subgraphs as well as forbidden substructures - chordless cycles and so-called asteroidal triples.

\footnotetext{
${ }^{*}$ Corresponding author

Email addresses: mathew@imsc.res.in (Mathew Francis), pavol@sfu.ca (Pavol Hell), j.stacho@warwick.ac.uk (Juraj Stacho)

${ }^{1}$ MF partially supported by the grant ANR-09-JCJC-0041.

${ }^{2} \mathrm{PH}$ partially supported by the author's NSERC Discovery Grant.

${ }^{3}$ JS gratefully acknowledges support from EPSRC, grant EP/I01795X/1.
} 
This result is the main motivation of our paper wherein we seek to describe analogous forbidden substructures for circular-arc graphs.

We remark in passing that, besides interval graphs, there are other subcases of circular-arc graphs that have already been characterized by the absence of simple obstructions. Namely, unit circular-arc graphs and proper circular-arc graphs in [16], chordal proper circular-arc graphs in [1], cobipartite circular-arc graphs in [13] and later in [6] (using so-called edge-asteroids), and Helly circular-arc graphs within circular-arc graphs in [12] (using so-called obstacles).

More recently, in [2], the authors gave forbidden induced subgraph characterizations for $P_{4}$-free circulararc graphs, diamond-free circular-arc graphs, paw-free circular-arc graphs, and most relevant for this paper, they characterized claw-free chordal circular-arc graphs. Our results (namely Theorem 4) may be seen as complementing their work, since in this regard we give a forbidden induced subgraph characterization of $\overline{K_{5}}$-free chordal circular-arc graphs.

\section{Blocking quadruple}

To build intuition, we start by recalling the definition of asteroidal triple. We say that a vertex $x$ misses a path $P$ in $G$ if $x$ has no neighbour on $P$.

Vertices $x, y, z$ form an asteroidal triple of $G$ if between any two of them, there is a path in $G$ missed by the third vertex. It is easy to see that an interval graph cannot have an asteroidal triple [11].

We say that vertices $x, y$ avoid vertices $z, w$ in $G$ if there exists an $x y$-path missed by both $z$ and $w$, or there exists a $z w$-path missed by both $x$ and $y$.

We say that vertices $x, y, z, w$ form a blocking quadruple (BQ) of $G$ if any two of them avoid the remaining two. Namely, if $x, y$ avoid $z, w$, if $x, z$ avoid $y, w$, and if $x, w$ avoid $y, z$.

Lemma 1. If $G$ is a circular-arc graph, then $G$ has no blocking quadruple.

To see this, observe that a BQ is always an independent set of size four. Now, suppose that $G$ has a circular-arc representation and the arcs representing vertices $x, y, z, w$ appear in this circular order. Then no path between $x$ and $z$ can be missed by both $y$ and $w$, and no path between $y$ and $w$ can be missed by $x$ and $z$. In other words, the vertices $x, z$ do not avoid $y, w$.

Let us now discuss various forms of blocking quadruples that one may encounter in graphs. One class of such examples arises from asteroidal triples: if $a, b, c$ form an asteroidal triple of $G$ and $d$ is a vertex of degree zero in $G$, then $a, b, c, d$ is a blocking quadruple. This can be seen in the first three graphs in Figure 1. Other ways of extending an asteroidal triple to a BQ are also illustrated in Figure 1. The vertices $a, b, c$ in

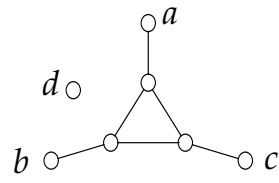

a)

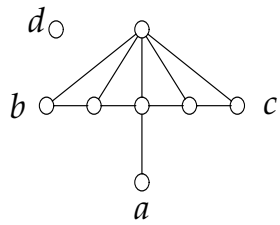

b)

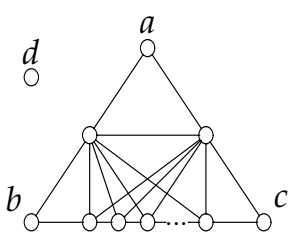

c)

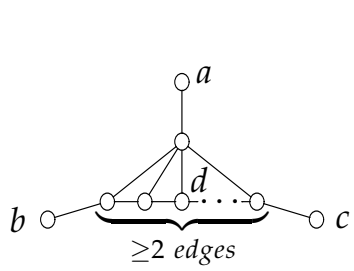

d)

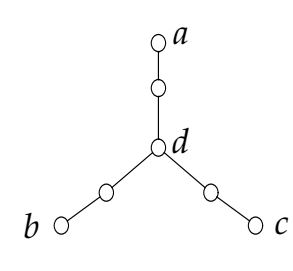

e)

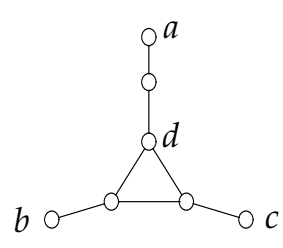

f)

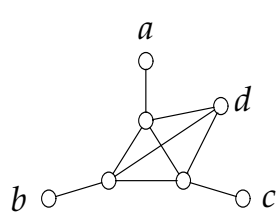

g)

Figure 1: Forbidden induced subgraph characterization of chordal graphs with no BQs. 
each of the graphs in the second row form an asteroidal triple while the vertices $a, b, c, d$ form a blocking quadruple. For chordal graphs, these are all possible forms of BQs (see Theorem 3).
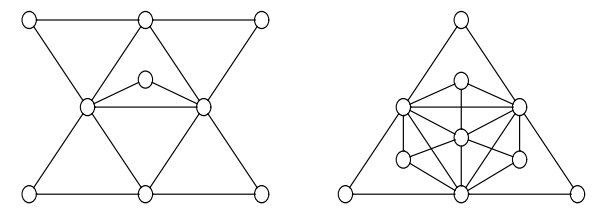

Figure 2: Some minimal chordal non-circular-arc graphs with no BQs.

Unlike these examples, the two chordal graphs in Figure 2 do not contain blocking quadruples, and yet they are not circular-arc graphs. Thus the absence of blocking quadruples is not sufficient to guarantee that a (chordal) graph is a circular-arc graph. However, in some cases, it may be sufficient.

For instance, a result of [2] (Corollary 15) can be restated as follows.

Lemma 2. A claw-free chordal graph is a circular-arc graph iff it has no BQ.

We prove a similar statement for chordal graphs of independence number at most four (see Theorem 4). The absence of BQs therefore gives us a simple and uniform forbidden structure characterization of these classes, as opposed to more common forbidden induced subgraph characterizations $[1,2,12,16]$.

\section{Main results}

In this section, we summarize the main theorems of this paper.

In the first theorem, we describe all minimal forbidden induced subgraphs characterizing chordal graphs with no BQs. These are the graphs depicted in Figure 1.

Theorem 3. If $G$ is chordal, then the following are equivalent.

(i) G contains a blocking quadruple.

(ii) G contains an induced subgraph isomorphic to a graph in Figure 1.

In fact, the theorem holds for the more general class of nearly chordal graphs (a graph class defined in [4] generalizing both chordal and circular-arc graphs).

In the second theorem, we show that the absence of BQs is necessary and sufficient for a chordal graph of independence number $\alpha(G) \leq 4$ to be a circular-arc graph.

Theorem 4. If $G$ is chordal and $\alpha(G) \leq 4$, the following are equivalent.

(i) $G$ is a circular-arc graph.

(ii) G contains no blocking quadruple.

The theorem fails for chordal graphs $G$ with $\alpha(G) \geq 5$ as Figure 2 shows.

Notice that this theorem implies that every chordal graph $G$ with $\alpha(G) \leq 3$ is a circular-arc graph (as any blocking quadruple is necessarily an independent set of size four). In contrast, it is known that every chordal graph $G$ with $\alpha(G) \leq 2$ is an interval graph (an asteroidal triple is also an independent set), and in fact, a proper interval graph (claw contains an independent set of size three).

\section{Proof of Theorem 3}

We prove a slightly more general statement (see Theorem 5 below).

A graph $G$ is a nearly chordal graph [4] if for each $v \in V(G)$, the graph $G-N[v]$ is a chordal graph. A graph $G$ is a nearly interval graph if for each $v \in V(G)$, the graph $G-N[v]$ is an interval graph. Clearly, every chordal graph is nearly chordal. 
Theorem 5. If $G$ is nearly chordal, then the following are equivalent.

(i) G contains a blocking quadruple.

(ii) G contains an induced subgraph isomorphic to a graph in Figure 1.

The proof of this theorem is split into the following two claims.

Lemma 6. Let $G$ be a nearly chordal graph. If $G$ is not a nearly interval graph, then $G$ contains one of the graphs in Figure 1 as an induced subgraph.

Proof. Suppose that $G$ is not a nearly interval graph. Then there exists a vertex $v \in V(G)$ such that $G-N[v]$ is not an interval graph. Since $G$ is a nearly chordal graph, we have that $G-N[v]$ is a chordal graph. Thus $G-N[v]$ contains as an induced subgraph one of the graphs a)-e) shown in Figure 3 [11].

If $G-N[v]$ contains the graphs d) or e) from Figure 3, then so does $G$ and the two graphs are also in Figure 1, namely d) and e), respectively. So we may assume that $G-N[v]$ contains one of the graphs a)-c) shown in Figure 3, which together with $v$ yields an induced subgraph of $G$ that is one of the graphs a)-c) shown in Figure 1. This concludes the proof.

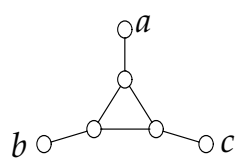

a)

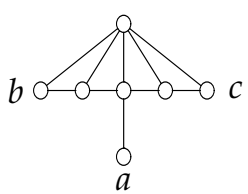

b)

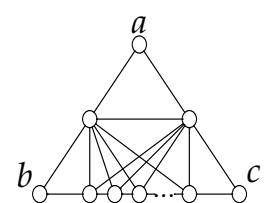

c)

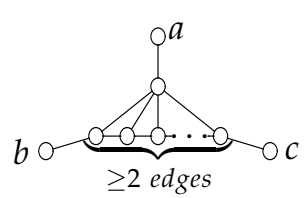

d)

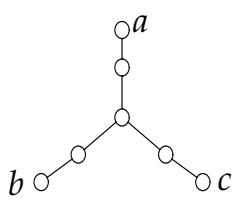

e)

Figure 3: All chordal minimal forbidden induced subgraphs of interval graphs.

Lemma 7. Let $G$ be a nearly interval graph. If $G$ has a blocking quadruple, then $G$ contains one of the graphs in Figure 1 as an induced subgraph.

Proof. Suppose that $G$ contains a blocking quadruple $a, b, c, d$. In particular, this implies that $G$ contains an $a b$-path missed by both $c$ and $d$, or a $c d$-path missed by both $a$ and $b$. Similarly, we have that $G$ contains an $a c$-path missed by $b, d$, or a $b d$-path missed by $a, c$.

By symmetry between $a, b, c, d$, we may assume without loss of generality that $G$ contains an $a b$-path $P_{b}$ missed by $c, d$ and an $a c$-path $P_{c}$ missed by $b, d$. (If not we replace $a$ by one of $b, c, d$ to satisfy this.) If $G$ also contains a $b c$-path missed by $a$ and $d$, then the vertices $a, b, c$ form an asteroidal triple in $G-N[d]$. Thus $G-N[d]$ is not an interval graph, contradicting our assumption that $G$ is a nearly interval graph.

We therefore conclude that every $b c$-path of $G$ contains a neighbour of $a$ or $d$. Since $a, b, c, d$ is a blocking quadruple, this implies that $G$ contains an $a d$-path $P_{d}$ missed by both $b$ and $c$. Moreover, since $G$ is a nearly interval graph, we have that every $b d$-path contains a neighbour of $a$ or $c$, and every $c d$-path contains a neighbour of $a$ or $b$. (Otherwise $G-N[c]$ or $G-N[b]$ is not an interval graph, providing a similar contradiction as above.) For later use, we summarize these observations as follows.

$\langle 1\rangle$ Every bc-path of $G$ contains a neighbour of a or $d$, every bd-path contains a neighbour of a or $c$, and every $c d$-path contains a neighbour of $a$ or $b$.

Now, without loss of generality, we shall assume that the quadruple $a, b, c, d$ and the paths $P_{b}, P_{c}, P_{d}$ were chosen so that $\left|P_{b}\right|+\left|P_{c}\right|+\left|P_{d}\right|$ is smallest possible. (If not, we simply replace $a, b, c, d$ or paths $P_{b}, P_{c}, P_{d}$ by a different quadruple or paths where the value is smaller and repeat the above.) The minimality of this choice implies that $P_{b}$ is a shortest $a b$-path in $G-N[c]-N[d]$. Similarly, $P_{c}$ is a shortest $a c$-path in $G-N[b]-N[d]$, and $P_{d}$ is a shortest $a d$-path in $G-N[b]-N[c]$. In particular, the three paths are induced paths of $G$.

Observe that the paths $P_{b}, P_{c}, P_{d}$ share at least one vertex, namely $a$. We prove that the minimality of our choice guarantees that $a$ is, in fact, the only vertex that these paths share. This is proved as follows. 
$\langle 2\rangle P_{b} \cap P_{c} \cap P_{d}=\{a\}$.

Suppose that $P_{b} \cap P_{c} \cap P_{d} \neq\{a\}$. Let $x$ be the vertex on $P_{b}$ such that $x \in P_{c} \cap P_{d}$ and no internal vertex of the subpath of $P_{b}$ between $x$ and $b$ is in $P_{c} \cap P_{d}$. Clearly, $x$ is well-defined, since $a \in P_{b} \cap P_{c} \cap P_{d}$. Moreover, we have $x \neq a$ by the choice of $x$ and the fact that $P_{b} \cap P_{c} \cap P_{d} \neq\{a\}$. Denote by $P_{b}^{\prime}$ the subpath of $P_{b}$ between $x$ and $b$. Similarly, since $x \in P_{c} \cap P_{d}$, denote by $P_{c}^{\prime}$ the subpath of $P_{c}$ between $x$ and $c$, and by $P_{d}^{\prime}$ the subpath of $P_{d}$ between $x$ and $d$. Observe that $P_{b}^{\prime}$ is missed by both $c$ and $d$, since $P_{b}$ is. Similary, $P_{c}^{\prime}$ is missed by $b, d$ and $P_{d}^{\prime}$ is missed by $b, c$, since $P_{c}$, respectively, $P_{d}$ is. We deduce that $x, b, c, d$ is a blocking quadruple of $G$, and $\left|P_{b}^{\prime}\right|+\left|P_{c}^{\prime}\right|+\left|P_{d}^{\prime}\right|<\left|P_{b}\right|+\left|P_{c}\right|+\left|P_{d}\right|$, since $x \neq a$. But that contradicts the choice of $a, b, c, d$.

This proves $\langle 2\rangle$.

$\langle 3\rangle P_{b} \cap P_{c}=P_{b} \cap P_{d}=P_{c} \cap P_{d}=\{a\}$.

By symmetry, suppose without loss of generality that $P_{b} \cap\left(P_{c} \cup P_{d}\right) \neq\{a\}$. Recall that $a \in P_{b} \cap P_{c} \cap P_{d}$. Let $x$ be the vertex of $P_{b}$ such that $x \in P_{c}$ and no internal vertex of the subpath of $P_{b}$ between $x$ and $b$ is in $P_{c}$. Let $y$ be the vertex of $P_{b}$ such that $y \in P_{d}$ and no internal vertex of the subpath of $P_{b}$ between $y$ and $b$ is in $P_{d}$.

Suppose that $x=y$. If $x=y=a$, then we conclude $P_{b} \cap P_{c}=\{a\}$ and $P_{b} \cap P_{d}=\{a\}$ by the choice of $x$ and $y$. However, we assume that $P_{b} \cap\left(P_{c} \cup P_{d}\right) \neq\{a\}$. Thus $x=y \neq a$ in which case we contradict $\langle 2\rangle$, since $x \in P_{b} \cap P_{c}$ and $y \in P_{b} \cap P_{d}$. Therefore, we must conclude that $x \neq y$.

Now, by symmetry, we shall assume without loss of generality that $x$ belongs to the subpath of $P_{b}$ between $y$ and $b$. Denote by $P_{b}^{\prime}$ the subpath of $P_{b}$ between $x$ and $b$, and by $P_{b}^{y x}$ the subpath of $P_{b}$ between $y$ and $x$. Since $x \in P_{c}$, denote by $P_{c}^{\prime}$ the subpath of $P_{c}$ between $x$ and $c$. Also, since $y \in P_{d}$, denote by $P_{d}^{y}$ the subpath of $P_{d}$ between $d$ and $y$, and define $P_{d}^{\prime}=P_{d}^{y} \cup P_{b}^{y x}$. Note that $P_{d}^{\prime}$ is a path between $d$ and $x$, since $P_{b}^{y x} \cap P_{d}=\{y\}$ by the choice of $y$. Further, observe that both $P_{b}^{\prime}$ and $P_{b}^{y x}$ are missed by $c$ and $d$, since $P_{b}$ is. Similarly, $P_{c}^{\prime}$ is missed by both $b$ and $d$, since $P_{c}$ is. Further, note that $x b \notin E(G)$, since $x \in P_{c}$ and $P_{c}$ is missed by $b$. This implies that $P_{b}^{y x}$ is missed by $b$, since $P_{b}$ is an induced path. Therefore, $P_{d}^{\prime}$ is missed by both $b$ and $c$, since both $P_{d}$ and $P_{b}^{y x}$ are. This shows that $x, b, c, d$ is a blocking quadruple. However, we observe that $a \neq x$, since $x \neq y$ and $x$ belongs to the subpath of $P_{b}$ between $y$ and $b$.

Thus $\left|P_{c}^{\prime}\right|<\left|P_{c}\right|$ which implies $\left|P_{b}^{\prime}\right|+\left|P_{c}^{\prime}\right|+\left|P_{d}^{\prime}\right|=\left|P_{b}^{\prime}\right|+\left|P_{c}^{\prime}\right|+\left|P_{b}^{y x}\right|+\left|P_{d}^{y}\right|-1 \leq\left|P_{b}\right|+\left|P_{c}^{\prime}\right|+\left|P_{d}\right|<$ $\left|P_{b}\right|+\left|P_{c}\right|+\left|P_{d}\right|$ which contradicts our choice of the quadruple $a, b, c, d$. This proves $\langle 3\rangle$.

In the next claims, we analyze the edges between the paths $P_{b}, P_{c}, P_{d}$.

$\langle 4\rangle$ For every edge uv of $G$ such that $u, v \in P_{b} \cup P_{c} \cup P_{d}$, we have that $u \in N(a)$ or $v \in N(a)$ or $u v$ is an edge of one of the paths $P_{b}, P_{c}, P_{d}$.

Consider an edge $u v \in E(G)$ where $u, v \in P_{b} \cup P_{c} \cup P_{d}$. For contradiction, suppose that $u, v \notin N(a)$ and that $u v$ is not an edge of one of the paths $P_{b}, P_{c}, P_{d}$. By symmetry, we may assume without loss of generality that $u \in P_{b}$ and $v \in P_{c}$. Let $P_{b}^{u}$ denote the subpath of $P_{b}$ between $b$ and $u$, and let $P_{c}^{v}$ denote the subpath of $P_{c}$ between $v$ and $c$. Note that both $P_{b}^{u}$ and $P_{c}^{v}$ are missed by $a$, since $u, v \notin N(a)$ and both $P_{b}$ and $P_{c}$ are induced paths. Define $P^{\prime}=P_{b}^{u} \cup P_{c}^{v}$, and observe that $P^{\prime}$ is a path by $\langle 3\rangle$. Moreover, $P^{\prime}$ is missed by $d$, since both $P_{b}$ and $P_{c}$ are. Also, $P^{\prime}$ is missed by $a$, since both $P_{b}^{u}$ and $P_{c}^{v}$ are. But then $P^{\prime}$ is a $b c$-path missed by both $a$ and $d$, contradicting $\langle 1\rangle$. This proves $\langle 4\rangle$.

$\langle 5\rangle$ Suppose that $u \in P_{c} \cup P_{d}$ has a neighbour $v$ on $P_{b}$. Then $u$ is adjacent to every internal vertex of the subpath of $P_{b}$ between $a$ and $v$.

By symmetry (replacing $c$ by $d$ and vice-versa), we may assume without loss of generality that $u \in P_{c}$.

For contradiction, suppose that $u$ is not adjacent to some internal vertex of the subpath of $P_{b}$ between $a$ and $v$. This implies that $v \notin N(a)$, and hence, $u \in N(a)$ by $\langle 4\rangle$. From this we conclude that $u$ has distinct neighbours $x, y$ on $P_{b}$ (possibly $\{x, y\} \cap\{a, v\} \neq \varnothing$ ) such that $x, y$ are not consecutive on $P_{b}$ and no internal vertex of the subpath of $P_{b}$ between $x$ and $y$ is adjacent to $u$. Let $P_{b}^{x y}$ denote this subpath. Note that $P_{b}^{x y}$ is an induced path, since $P_{b}$ is. So, since $x, y$ are not consecutive on $P_{b}$, we have $\left|P_{b}^{x y}\right| \geq 3$. Further, recall that the only neighbours of $u$ on $P_{b}^{x y}$ are $x$ and $y$. From this we conclude that $C=P_{b}^{x y} \cup\{u\}$ induces in $G$ 
a cycle of length four or more. In particular, no vertex of $C$ is adjacent to $d$, since $C \subseteq P_{b} \cup P_{c}$ and both $P_{b}$ and $P_{c}$ are missed by $d$. Thus $C$ is an induced cycle of length four or more in $G-N[d]$, contradicting our assumption that $G$ is a nearly interval graph. This proves $\langle 5\rangle$.

Using the symmetry between the paths $P_{b}, P_{c}, P_{d}$, we may generalize this to the following statement.

$\left\langle 5^{+}\right\rangle$Suppose that $u \in P_{c} \cup P_{d}$ has a neighbour $v$ on $P_{b}$. Then $u$ is adjacent to every internal vertex of the subpath of $P_{b}$ between $a$ and $v$.

Suppose that $u \in P_{b} \cup P_{d}$ has a neighbour $v$ on $P_{c}$. Then $u$ is adjacent to every internal vertex of the subpath of $P_{c}$ between $a$ and $v$.

Suppose that $u \in P_{b} \cup P_{c}$ has a neighbour $v$ on $P_{d}$. Then $u$ is adjacent to every internal vertex of the subpath of $P_{d}$ between $a$ and $v$.

〈6) Suppose that $\left|P_{b}\right| \geq 4$. If there are edges between the internal vertices of $P_{b}$ and $P_{c}$, then there are no edges between the internal vertices of $P_{b}$ and $P_{d}$.

For contradiction, suppose that some internal vertex $v$ of $P_{b}$ has a neighbour $x \notin\{a, c\}$ on $P_{c}$, and some internal vertex $w$ of $P_{b}$ (possibly $v=w$ ) has a neighbour $y \notin\{a, d\}$ on $P_{d}$. Let $u$ denote the neighbour of $a$ on $P_{b}$. From $\left\langle 5^{+}\right\rangle$applied to $x$ and $v$, we conclude that $u x \in E(G)$. Similarly, using $\left\langle 5^{+}\right\rangle$applied to $y$ and $w$, we obtain $u y \in E(G)$.

Now, recall that $\left|P_{b}\right| \geq 4$. This implies that $u b \notin E(G)$, since $P_{b}$ is an induced path. Let $P_{b}^{u}$ be the subpath of $P_{b}$ between $u$ and $b$, let $P_{c}^{x}$ be the subpath of $P_{c}$ between $x$ and $c$, and let $P_{d}^{y}$ be the subpath of $P_{d}$ between $y$ and $d$. Define $P_{b}^{\prime}=P_{b}^{u}, P_{c}^{\prime}=\{u\} \cup P_{c}^{x}$, and $P_{d}^{\prime}=\{u\} \cup P_{d}^{y}$. Clearly, both $P_{c}^{\prime}$ and $P_{d}^{\prime}$ are paths of $G$, since $u \notin P_{c} \cup P_{d}$ by $\langle 3\rangle$. Moreover, $u c, u d \notin E(G)$ because $u \in P_{b}$ and $P_{b}$ is missed by both $c$ and $d$.

Observe now that $P_{b}^{\prime}$ is missed by $c$ and $d$, since $P_{b}$ is. Moreover, $P_{c}^{\prime}$ is missed by both $b$ and $d$, since $u b, u d \notin E(G)$ and since $P_{c}$ is missed by both $b$ and $d$. Similarly, $P_{d}^{\prime}$ is missed by both $b$ and $c$, since $u b, u c \notin E(G)$ and since $P_{d}$ is missed by both $b$ and $c$. Thus we conclude that $u, b, c, d$ is a blocking quadruple of $G$. In particular, we have $\left|P_{c}^{\prime}\right| \leq\left|P_{c}\right|$ and $\left|P_{d}^{\prime}\right| \leq\left|P_{d}\right|$, since $a \notin\{x, y\}$. Also, $\left|P_{b}^{\prime}\right|<\left|P_{b}\right|$ by the definition of $u$. So we conclude that $\left|P_{b}^{\prime}\right|+\left|P_{c}^{\prime}\right|+\left|P_{d}^{\prime}\right|<\left|P_{b}\right|+\left|P_{c}\right|+\left|P_{d}\right|$, which contradicts the minimality of our choice of $a, b, c, d$ and paths $P_{b}, P_{c}, P_{d}$. This proves $\langle 6\rangle$.

A symmetric argument (using $P_{c}$ or $P_{d}$ in place of $P_{b}$ ) yields the following.

$\left\langle 6^{+}\right\rangle$Suppose that $\left|P_{b}\right| \geq 4$. If there are edges between the internal vertices of $P_{b}$ and $P_{c}$, then there are no edges between the internal vertices of $P_{b}$ and $P_{d}$.

Suppose that $\left|P_{c}\right| \geq 4$. If there are edges between the internal vertices of $P_{c}$ and $P_{b}$, then there are no edges between the internal vertices of $P_{c}$ and $P_{d}$.

Suppose that $\left|P_{d}\right| \geq 4$. If there are edges between the internal vertices of $P_{d}$ and $P_{b}$, then there are no edges between the internal vertices of $P_{d}$ and $P_{c}$.

(7) Suppose that $\left|P_{b}\right| \geq 4$ and $\left|P_{c}\right| \geq 4$. Then there are no edges between the internal vertices of $P_{b}$ and $P_{c}$.

For contradiction, suppose that an internal vertex $u$ of $P_{b}$ is adjacent to an internal vertex $v$ of $P_{c}$. By $\langle 4\rangle$, we conclude $u \in N(a)$ or $v \in N(a)$. By symmetry, we may assume without loss of generality that $u \in N(a)$. This implies $u b \notin E(G)$, since $P_{b}$ is an induced path and $\left|P_{b}\right| \geq 4$. Since $v$ is an internal vertex of $P_{c}$ and $c$ has no neighbour on $P_{b}$, we conclude that there exist consecutive vertices $x, y$ on $P_{c}$ such that $x \neq a$, $u x \in E(G), u y \notin E(G)$, and $y$ belongs to the subpath of $P_{c}$ between $x$ and $c$ (possibly $y=c$.) Note that $y$ has no neighbours on $P_{d}$, since otherwise we contradict $\left\langle 6^{+}\right\rangle$for $P_{c}$. (For this, recall that $d$ misses $P_{c}$, that $\left|P_{c}\right| \geq 4$, that $x \in P_{c}$ is adjacent to $u \in P_{b}$, and that $a y \notin E(G)$ because $P_{c}$ is an induced path and $x$ belongs to the subpath of $P_{c}$ between $a$ and $y$.)

Let $P_{b}^{\prime}$ denote the subpath of $P_{b}$ between $u$ and $b$. Let $P_{y}^{\prime}=\{u, x, y\}$ and $P_{d}^{\prime}=\{u\} \cup P_{d}$. Note that $P_{b}^{\prime}, P_{y}^{\prime}, P_{d}^{\prime}$ are paths of $G$, since $u \notin P_{d}$ by $\langle 3\rangle$. Observe that $P_{y}^{\prime}$ is missed by $d$, since $P_{y}^{\prime} \subseteq P_{b} \cup P_{c}$ and both $P_{b}$ and $P_{c}$ are missed by $d$. Also, $P_{y}^{\prime}$ is missed by $b$, since $P_{c}$ is missed by $b$ and since $u b \notin E(G)$. The fact that $u b \notin E(G)$ also implies that $P_{d}^{\prime}$ is missed by $b$, since $P_{d}$ is. Moreover, $P_{d}^{\prime}$ is missed by $y$, since $u y \notin E(G)$ and $y$ has no neighbour on $P_{d}$ as observed earlier. Finally, note that $y \notin N(a)$ because $x \neq a$ and $P_{c}$ is an 
induced path. Thus we conclude that $y$ has no neighbour on $P_{b}$, since by $\langle 4\rangle$ the only possible neighbour of $y$ on $P_{b}$ is $u$, because $y \notin N(a)$, but we have $u y \notin E(G)$ by the choice of $y$. Thus $P_{b}^{\prime}$ is missed by $y$, since $P_{b}^{\prime}$ is a subpath of $P_{b}$. Moreover, $P_{b}^{\prime}$ is missed by $d$, since $P_{b}$ is. This shows that $u, b, y, d$ is a blocking quadruple. However, $\left|P_{b}^{\prime}\right|+\left|P_{y}^{\prime}\right|+\left|P_{d}^{\prime}\right|<\left|P_{b}\right|+\left|P_{c}\right|+\left|P_{d}\right|$, since $\left|P_{y}^{\prime}\right|=3<4 \leq\left|P_{c}\right|$ while $\left|P_{b}^{\prime}\right|+\left|P_{d}^{\prime}\right|=\left|P_{b}\right|+\left|P_{d}\right|$. Thus we contradict the minimality of $a, b, c, d$. This proves $\langle 7\rangle$.

A symmetric argument (using $P_{b}, P_{d}$ or $P_{c}, P_{d}$ in place of $P_{b}, P_{c}$ ) yields:

$\left\langle 7^{+}\right\rangle$Suppose that $\left|P_{b}\right| \geq 4$ and $\left|P_{c}\right| \geq 4$. Then there are no edges between the internal vertices of $P_{b}$ and $P_{c}$. Suppose that $\left|P_{b}\right| \geq 4$ and $\left|P_{d}\right| \geq 4$. Then there are no edges between the internal vertices of $P_{b}$ and $P_{d}$. Suppose that $\left|P_{c}\right| \geq 4$ and $\left|P_{d}\right| \geq 4$. Then there are no edges between the internal vertices of $P_{c}$ and $P_{d}$.

$\langle 8\rangle$ Let $v$ be the neighbour of $b$ on $P_{b}$. Then $v$ has a neighbour on $P_{c}$ or $P_{d}$.

For contradiction, suppose that no vertex of $P_{c} \cup P_{d}$ is adjacent to $v$. Note that this implies $v \neq a$ and va $\notin E(G)$. Let $P_{v}$ denote the subpath of $P_{b}$ betwenn $a$ and $v$. Clearly, $P_{v}$ is missed by both $c$ and $d$, since $P_{b}$ is. Moreover, $P_{c}$ and $P_{d}$ are missed by $v$, since no vertex of $P_{c} \cup P_{d}$ is adjacent to $v$. Recall that $P_{c}$ is missed by $d$ and that $P_{d}$ is missed by $c$. Thus $a, v, c, d$ is a blocking quadruple. However, since $b \notin P_{v}$, we have $\left|P_{v}\right|<\left|P_{b}\right|$, and hence, $\left|P_{v}\right|+\left|P_{c}\right|+\left|P_{d}\right|<\left|P_{b}\right|+\left|P_{c}\right|+\left|P_{d}\right|$, which contradicts the minimality of $a, b, c, d$.

This proves $\langle 8\rangle$.

Again, by symmetry (between $b, c$, and $d$ ), we can conclude the following.

$\left\langle 8^{+}\right\rangle$The neighbour of $b$ on $P_{b}$ has a neighbour on $P_{c}$ or $P_{d}$.

The neighbour of $c$ on $P_{c}$ has a neighbour on $P_{b}$ or $P_{d}$.

The neighbour of $d$ on $P_{d}$ has a neighbour on $P_{b}$ or $P_{c}$.

The last claim in this series of statements is as follows.

$\langle 9\rangle$ If $\left|P_{b}\right| \geq 4$, then there are edges between the internal vertices of $P_{c}$ and $P_{d}$.

Assume there are no edges between the internal vertices of $P_{c}$ and $P_{d}$. Let $u$ be the neighbour of $b$ on $P_{b}$. Let $x$ be the neighbour of $a$ on $P_{b}$. Clearly, $u \neq a$ and $u a, x b \notin E(G)$, since $P_{b}$ is an induced path, and $\left|P_{b}\right| \geq 4$.

By $\left\langle 8^{+}\right\rangle, u$ has a neighbour on $P_{c}$ or $P_{d}$. By symmetry, we may assume, without loss of generality, that $u$ has a neighbour $v$ on $P_{c}$. Note that $v \neq a$, since $u a \notin E(G)$. Also, $v \neq c$, since $c$ misses the path $P_{b}$ and $u \in P_{b}$. Thus $v$ is an internal vertex of $P_{c}$. From $\left\langle 5^{+}\right\rangle$, we deduce that $v x \in E(G)$, since $v u \in E(G)$ and $x$ lies on the subpath of $P_{b}$ between $a$ and $u$. By $\left\langle 6^{+}\right\rangle$, there are no edges between the internal vertices of $P_{b}$ and $P_{d}$, since $u \in P_{b}$ is adjacent to $v \in P_{c}$ which are both internal vertices of the respective paths. For the same reason, we have, by $\left\langle 7^{+}\right\rangle$, that $\left|P_{c}\right|=3$, namely, that $P_{c}=\{a, v, c\}$,

Now, let $w$ denote the neighbour of $a$ on $P_{d}$. Let $P_{c}^{\prime}=\{x, v, c\}$, let $P_{w}^{\prime}=\{x, a, w\}$, and let $P_{b}^{\prime}$ denote the subpath of $P_{b}$ between $x$ and $b$. Note that $v w \notin E(G)$, since $v \in P_{c}, w \in P_{d}$, and we assume that there are no edges between the internal vertices of $P_{c}$ and $P_{d}$. So, we conclude that $P_{c}^{\prime}$ and $P_{b}^{\prime}$ are missed by $w$, since $v w \notin E(G)$ and $c$ misses $P_{d}$, while $w \in P_{d}$, there are no edges between the internal vertices of $P_{b}$ and $P_{d}$, and $b$ misses $P_{d}$. Moreover, $P_{b}^{\prime}$ is missed by $c$, since $P_{b}$ is. Similarly, $P_{c}^{\prime}$ and $P_{w}^{\prime}$ are missed by $b$, since $P_{c}$ and $P_{d}$ are, and since $x b \notin E(G)$. Likewise, $P_{w}^{\prime}$ is missed by $c$, since $P_{w}^{\prime} \subseteq P_{b} \cup P_{d}$ and $c$ misses both $P_{b}$ and $P_{d}$. This shows that $x, b, c, w$ is a blocking quadruple, and we have $\left|P_{c}^{\prime}\right|=\left|P_{c}\right|$, and $\left|P_{b}^{\prime}\right|<\left|P_{b}\right|$ while $\left|P_{w}^{\prime}\right| \leq\left|P_{d}\right|$, since $w$ is an internal vertex of $P_{d}$. So $\left|P_{b}^{\prime}\right|+\left|P_{c}^{\prime}\right|+\left|P_{w}^{\prime}\right|<\left|P_{b}\right|+\left|P_{c}\right|+\left|P_{d}\right|$ contradicting our choice of $a, b, c, d$.

This proves $\langle 9\rangle$.

As before, we deduce from this (by symmetry) the following.

$\left\langle 9^{+}\right\rangle$If $\left|P_{b}\right| \geq 4$, there are edges between the internal vertices of $P_{c}$ and $P_{d}$.

If $\left|P_{c}\right| \geq 4$, there are edges between the internal vertices of $P_{b}$ and $P_{d}$.

If $\left|P_{d}\right| \geq 4$, there are edges between the internal vertices of $P_{b}$ and $P_{c}$.

We are finally ready to find an induced subgraph in $G$ isomorphic to one of the configurations in Figure 1. To start, note that $a, b, c, d$ are pairwise non-adjacent, since they form a blocking quadruple. This implies that the paths $P_{b}, P_{c}, P_{d}$ have each at least three vertices. 
Let $u$ denote the neighbour of $b$ on $P_{b}$, let $v$ denote the neighbour of $c$ on $P_{c}$, and let $w$ denote the neighbour of $d$ on $P_{d}$. Clearly, $a \notin\{u, v, w\}$.

Suppose first that one of the three paths contains at least four vertices. Without loss of generality (by the symmetry between the three paths), assume that $\left|P_{b}\right| \geq 4$. This implies that $u a \notin E(G)$, since $P_{b}$ is an induced path. Further, by $\left\langle 8^{+}\right\rangle$, note that $u$ has a neighbour on $P_{c} \cup P_{d}$. Without loss of generality (again by symmetry), assume that $u$ has a neighbour on $P_{c}$.

This implies, by $\left\langle 7^{+}\right\rangle$, that $\left|P_{c}\right|=3$. Namely, we have that $P_{c}=\{a, v, c\}$. Moreover, by $\left\langle 6^{+}\right\rangle$, there are no edges between the internal vertices of $P_{b}$ and $P_{d}$, since $\left|P_{b}\right| \geq 4$ and $u \in P_{b}$ has a neighbour on $P_{c}$. In addition, recall that $c$ misses the path $P_{b}$, that $u \in P_{b}$, and that $u a \notin E(G)$. Thus, since $u$ has a neighbour on $P_{c}$, we conclude that $u v \in E(G)$. This implies, by $\left\langle 5^{+}\right\rangle$, that $v$ is adjacent to all internal vertices on $P_{b}$.

Suppose that $v w \notin E(G)$. If $\left|P_{d}\right| \geq 4$, then $w c, w a \notin E(G)$, since $c$ misses $P_{d}$ and since $P_{d}$ is an induced path. Thus $w$ has no neighbours on $P_{c}$, and also no neighbours on $P_{b}$, since $b$ misses $P_{d}$ and there are no edges between the internal vertices of $P_{b}$ and $P_{d}$. This contradicts $\left\langle 8^{+}\right\rangle$. Therefore $\left|P_{d}\right|=3$ in which case $v$, $w$ are the only internal vertices of $P_{c}$ and $P_{d}$, respectively. But we assume $v w \notin E(G)$, contradicting $\left\langle 9^{+}\right\rangle$.

We must therefore conclude that $v w \in E(G)$. So, by $\left\langle 5^{+}\right\rangle$, the vertex $v$ is adjacent to all internal vertices of $P_{d}$. Recall that $v$ is also adjacent to all internal vertices of $P_{b}$, and there are no edges between the internal vertices of $P_{b}$ and $P_{d}$. Thus, since the paths $P_{b}, P_{c}, P_{d}$ are induced, we conclude that the union $P_{b} \cup P_{c} \cup P_{d}$ of the three paths induces in $G$ the graph d) in Figure 1. This completes the case when one of the three paths has four or more vertices.

It remains to discuss the case when each of the three paths $P_{b}, P_{c}, P_{d}$ has exactly three vertices. Namely, we have $P_{b}=\{a, u, b\}, P_{c}=\{a, v, c\}$, and $P_{d}=\{a, w, d\}$. In this case, we show that their union $P_{b} \cup P_{c} \cup P_{d}$ induces in $G$ one of the graphs d)-g) in Figure 1. In particular, if $u v, u w, v w \notin E(G)$, then the paths induce the graph e) in Figure 1, while if $u v, u w, v w \in E(G)$, the paths induce the graph g) in Figure 1. Similarly, if exactly one of $u v, u w, v w$ is in $E(G)$, then the paths induce the graph $f$ ) in Figure 1, while if exactly two of $u v, u w, v w$ are in $E(G)$, the paths induce the graph d) in Figure 1 where the path labelled " $\geq 2$ edges" has exactly 2 edges. This exhausts all possibilities and concludes the proof of Lemma 7.

Finally, to prove Theorem 5, suppose that $G$ is a nearly chordal graph. If $G$ contains a subgraph isomorphic to one of the graphs in Figure 1, then $G$ contains a blocking quadruple, as each of the graphs in Figure 1 contains one on the vertices labelled as $a, b, c, d$. Conversely, if $G$ does not contain as an induced subgraph any of the graphs in Figure 1, then $G$ is a nearly interval graph by Lemma 6 . So $G$ contains no blocking quadruple by Lemma 7. This proves the equivalence of (i) and (ii) of Theorem 5.

\section{Proof of Theorem 4}

Before the proof itself, we need to introduce some useful notions. We shall describe a particular general construction of circular arcs, corresponding to the vertices of a given chordal graph $G$, which will be based on a clique tree $T$ of $G$ and its planar drawing. Intuitively, this operation will correspond to cutting out the drawing of $T$ from the plane and stretching the resulting hole to a disc. The cliques of $T$ will appear, possibly multiple times, in the cyclic sequence of cliques on the boundary of this disc. We observe that the same sequence of cliques can be obtained by a suitable depth-first traversal of $T$ or an Euler tour of $T$ (when considered as a symmetric digraph). For convenience, we shall use the latter.

We then use this cyclic sequence to generate a family of circular-arcs. The construction will produce circular arcs for all vertices of $G$, but we will not be able to guarantee that the intersections of these arcs correspond to the edges in $G$. To ensure this, we introduce a set of conditions that will suffice to imply that the intersection graph of the arcs will indeed be $G$.

Finally, to prove Theorem 4, we will explain how to choose a clique tree of $G$ and an appropriate planar drawing of it (i.e., an appropriate Euler tour) so that these conditions are fulfilled.

\subsection{Preliminaries}

Let $G$ be a chordal graph. A clique of $G$ is a set of pairwise adjacent vertices. A maximal clique of $G$ is a clique that is not contained in a larger clique of $G$. 
A clique tree of $G$ is a tree $T$ whose vertices are the maximal cliques of $G$ such that for all pairs of maximal cliques $C, C^{\prime}$ of $G$, if $C^{\prime \prime}$ is a maximal clique on the path of $T$ between $C$ and $C^{\prime}$, then $C^{\prime \prime} \supseteq C \cap C^{\prime}$. Every chordal graph has a clique tree, and a graph is chordal if and only if it has a clique tree [7]. Note that a chordal graph can have multiple clique trees.

We shall assume that $G$ contains no universal vertex (vertex adjacent to all other vertices of $G$ ). This does not change any of the subsequent arguments as a circular-arc graph remains circular-arc on addition of universal vertices.

A clique cover of $G$ is a collection of maximal cliques of $G$ such that every vertex in $G$ belongs to at least one of the cliques in the clique cover.

The following lemmas are simple consequences of the respective definitions. We leave the details of their proofs to the reader.

Lemma 8. If $T$ is a clique tree of $G$, and $C$ is a leaf of $T$, then there exists a vertex $u \in V(G)$ such that $u$ is in $C$ and in no other clique in $V(T)$.

Lemma 9. If $\mathcal{Q}$ is a clique cover of $G$, and $T$ is a clique tree of $G$, then every leaf of $T$ is in $\mathcal{Q}$.

\subsection{Euler tour}

Let $T$ be a clique tree of $G$. Note that $T$ can be considered as a symmetric directed graph (where each edge is replaced by a pair of directed arcs with opposite directions). Then each vertex in $T$ has its in-degree equal to its out-degree. Thus if considered as such, $T$ is a connected Eulerian digraph, and hence, has an Euler tour (a walk visiting all edges of $T$ ).

We shall write $\mathcal{A}=A_{0}, A_{1}, \ldots, A_{k-1}, A_{0}$ for an Euler tour of $T$ visiting nodes $A_{0}, A_{1}, \ldots, A_{k-1}, A_{0}$ in this order. Note that nodes of $T$ may appear multiple times in $\mathcal{A}$ as different $A_{i}$ s. Nonetheless, every leaf of $T$ appears exactly once in $\mathcal{A}$. For $i, j \in\{0, \ldots, k-1\}$, we write $\mathcal{A}[i, j]$ to denote the circular subsequence $A_{i}, A_{i+1}, \ldots, A_{j}$ (indices mod $k$ ) of $\mathcal{A}$. In other words,

We write $\mathcal{A}(i, j)$ for $\mathcal{A}[i, j] \backslash\left\{A_{i}, A_{j}\right\}$.

$$
\mathcal{A}[i, j]= \begin{cases}A_{i}, A_{i+1}, \ldots, A_{j} & \text { if } i \leq j \\ A_{i}, \ldots, A_{k-1}, A_{0}, \ldots, A_{j} & \text { if } i>j\end{cases}
$$

Our main tool in proving Theorem 4 is the following lemma.

Lemma 10. Let $T$ be a clique tree of $G$, and let $\mathcal{A}=A_{0}, A_{1}, \ldots, A_{k-1}, A_{0}$ be an Euler tour of $T$. If there exists a mapping $\phi: V(G) \rightarrow\{0, \ldots, k-1\}$ such that

$(\star 0) u \in A_{\phi(u)}$ for each $u \in V(G)$,

and such that each $u v \in E(G)$ satisfies at least one of the following conditions:

$(\star 1) u x \in E(G)$ for every $x \in V(G)$ such that $A_{\phi(x)} \in \mathcal{A}(\phi(u), \phi(v))$,

$(\star 2) v x \in E(G)$ for every $x \in V(G)$ such that $A_{\phi(x)} \in \mathcal{A}(\phi(u), \phi(v))$,

$(\star 3)$ ux $\in E(G)$ for every $x \in V(G)$ such that $A_{\phi(x)} \in \mathcal{A}(\phi(v), \phi(u))$,

$(\star 4) \quad v x \in E(G)$ for every $x \in V(G)$ such that $A_{\phi(x)} \in \mathcal{A}(\phi(v), \phi(u))$,

then $G$ is a circular-arc graph.

Proof. In the subsequent text, all the subscript arithmetic is considered reduced modulo $k$.

We start with the following useful observation.

$\langle 10\rangle$ Let $u \in V(G)$. If $u$ is in $A_{i}$ and $A_{j}$ where $\mathcal{A}(i, j) \neq \varnothing$, and $u$ does not belong to any clique in $\mathcal{A}(i, j)$, then

(i) $A_{i}=A_{j}$

(ii) the path in $T$ from any clique in $\mathcal{A}[j, i]$ to any clique in $\mathcal{A}(i, j)$ passes through $A_{i}$, and

(iii) at least one of the cliques in $\mathcal{A}(i, j)$ is a leaf of $T$. 
Suppose $A_{i} \neq A_{j}$. Let $e$ be the edge of $T$ between $A_{i}$ and $A_{i+1}$. Note that $A_{i+1} \neq A_{j}$ because $\mathcal{A}(i, j) \neq \varnothing$ and because no clique in $\mathcal{A}(i, j)$ contains $u$ while $A_{j}$ does. Further note that, since $u$ is also in $A_{i}$, it is not possible that the edge $e$ is again traversed (in the reverse direction) somewhere between $A_{i+1}$ and $A_{j}$. This implies that $A_{i+1}$ lies on the path of $T$ between $A_{i}$ and $A_{j}$. However, this is impossible as $T$ is a clique tree, and both $A_{i}$ and $A_{j}$ contain $u$ while $A_{i+1}$ does not. This proves (i).

For (ii), we assume (i), i.e., that $A_{i}=A_{j}$, and first we show that also $A_{i+1}=A_{j-1}$. Let $\mathcal{M}$ denote the set of all the cliques that appear in $\mathcal{A}(i, j)$. As $\mathcal{A}(i, j)$ is a walk that goes through all the cliques in $\mathcal{M}$ and through no other clique, $\mathcal{M}$ induces a connected subgraph in $T$. Note that $A_{i}=A_{j}$ does not belong to $\mathcal{M}$ as it contains $u$ while no clique in $\mathcal{M}$ does, by our assumption. If $A_{i+1} \neq A_{j-1}$, then the path in $\mathcal{M}$ between $A_{i+1}$ and $A_{j-1}$ together with the edges $A_{i} A_{i+1}$ and $A_{i} A_{j-1}$ form a cycle in $T$ (recall that $A_{i}=A_{j}$ and $A_{i} \notin \mathcal{M}$ ), which is a contradiction. Therefore, $A_{i+1}=A_{j-1}$. Now, let $e$ again be the edge of $T$ between $A_{i}=A_{j}$ and $A_{i+1}=A_{j-1}$. The removal of the edge $e$ from $T$ results in two trees; let us denote them $T_{1}$ and $T_{2}$, and by symmetry, assume that $A_{i} \in V\left(T_{1}\right)$ while $A_{i+1} \in V\left(T_{2}\right)$. As $e$ is traversed in $\mathcal{A}$ exactly twice, once from $A_{i}$ to $A_{i+1}$ and once from $A_{j-1}$ to $A_{j}$, we conclude that the vertex set of $T_{1}$ consists precisely of the cliques appearing in $\mathcal{A}[j, i]$ while the vertices of $T_{2}$ are the cliques appearing in $\mathcal{A}(i, j)$. Note that, as $T$ is a tree, any path of $T$ between a vertex in $T_{1}$ and a vertex in $T_{2}$ must pass through the edge $e$. Therefore, so does any path of $T$ from a clique in $\mathcal{A}[j, i]$ to a clique in $\mathcal{A}(i, j)$. Any such path contains $A_{i}$, which proves (ii).

Finally, for (iii), recall $T_{2}$ from the previous paragraph, and note that the vertex set of $T_{2}$ consists of all cliques appearing in $\mathcal{A}(i, j)$. Thus, to prove (iii), it suffices to show that some vertex of $T_{2}$ is a leaf of $T$. If $T_{2}$ contains only one vertex, then this vertex is itself a leaf of $T$, and we are done. Otherwise, $T_{2}$ has at least two vertices, and so it has at least two leaves, at most one of which is incident to the edge $e$. Consequently any other leaf of $T_{2}$ is also a leaf of $T$, which implies (iii). This proves $\langle 10\rangle$.

Now, let $\phi: V(G) \rightarrow\{0, \ldots, k-1\}$ be a mapping satisfying the conditions of the lemma. Define circular $\operatorname{arcs}\left\{\mathcal{S}_{u}\right\}_{u \in V(G)}$ for the vertices of $G$ as follows. Let $\lambda_{0}, \lambda_{1}, \ldots, \lambda_{k-1}$ be $k$ distinct points on the circle, arranged in this clockwise order. For each vertex $u \in V(G)$, define indices $\ell_{u}$ and $r_{u}$ such that

- $A_{\ell_{u}}$ is the first clique that does not contain $u$ in

- $A_{r_{u}}$ is the first clique that does not contain $u$ in

$$
A_{\phi(u)-1}, A_{\phi(u)-2}, \ldots, A_{0}, A_{k-1}, A_{k-2}, \ldots, A_{\phi(u)+1}
$$

$$
A_{\phi(u)+1}, A_{\phi(u)+2}, \ldots, A_{k-1}, A_{0}, A_{1}, \ldots, A_{\phi(u)-1}
$$

Then define $\mathcal{S}_{u}$ to be the clockwise circular arc from $\lambda_{\ell_{u}+1}$ to $\lambda_{r_{u}-1}$.

We show that the arcs $\left\{\mathcal{S}_{u}\right\}_{u \in V(G)}$ constitute a circular-arc representation of $G$. Namely, we prove that for all vertices $u, v \in V(G)$, the arc $\mathcal{S}_{u}$ intersects the arc $\mathcal{S}_{v}$ if and only if $u v \in E(G)$. This will prove the lemma. We shall need the following property which can be deduced directly from the construction.

$\langle 11\rangle$ Each $u \in V(G)$ satisfies $\lambda_{\phi(u)} \in \mathcal{S}_{u}$, and $u \in A_{i}$ whenever $\lambda_{i} \in \mathcal{S}_{u}$.

$\langle 12\rangle$ If $\mathcal{S}_{u} \cap \mathcal{S}_{v} \neq \varnothing$ then $u v \in E(G)$.

Since $\mathcal{S}_{u} \cap \mathcal{S}_{v} \neq \varnothing$, let $i$ be such that $\lambda_{i} \in \mathcal{S}_{u} \cap \mathcal{S}_{v}$. This implies that $u, v \in A_{i}$ by $\langle 11\rangle$. Therefore $u v \in E(G)$, since $A_{i}$ is a clique of $G$. This proves $\langle 12\rangle$.

$\langle 13\rangle$ If $u v \in E(G)$, then $\mathcal{S}_{u} \cap \mathcal{S}_{v} \neq \varnothing$.

For contradiction, assume $\mathcal{S}_{u} \cap \mathcal{S}_{v}=\varnothing$. If $\phi(u)=\phi(v)$, then $\lambda_{\phi(u)} \in \mathcal{S}_{u} \cap \mathcal{S}_{v}$ by $\langle 11\rangle$, but this contradicts our assumption that $\mathcal{S}_{u} \cap \mathcal{S}_{v}=\varnothing$. Thus $\phi(u) \neq \phi(v)$.

By the assumptions of the lemma, one of the conditions $(\star 1)-(\star 4)$ is satisfied for the edge $u v$. Notice that the four conditions are the same up to reversal of $\mathcal{A}$ and exchanging $u$ and $v$. Indeed, it can be easily seen that reversing $\mathcal{A}$ will yield the same collection of circular-arcs up to some rotational symmetries. Therefore, we shall assume without loss of generality that $(\star 1)$ holds.

We claim that the cliques containing $u$ form a consecutive sequence in $\mathcal{A}[\phi(u), \phi(v)]$. Suppose not. Let $A_{i}$ be the first clique in $\mathcal{A}[\phi(u), \phi(v)]$ such that $u \notin A_{i}$ and let $A_{j}$ be the first clique in $\mathcal{A}[i, \phi(v)]$ that contains $u$. By $\langle 10\rangle$ (iii), this means that there is some clique $Q$ in $\mathcal{A}(i-1, j)$ that is a leaf of $T$ and does not contain $u$. 
As $Q$ is a leaf of $T$, by Lemma 8, there is a vertex $x$ that is in $Q$ but in no other clique in $V(T)$. But as $u \notin Q$, we have $u x \notin E(G)$. Also, as $Q$ is a leaf of $T$, it occurs exactly once in $\mathcal{A}$ and this occurrence is $A_{\phi(x)}$ by $(\star 0)$. So we have a vertex $x$ such that $A_{\phi(x)} \in \mathcal{A}(\phi(u), \phi(v))$ and $u x \notin E(G)$, which contradicts $(\star 1)$. Therefore, the cliques containing $u$ indeed form a consecutive sequence in $\mathcal{A}[\phi(u), \phi(v)]$. In other words, for every $A_{i} \in \mathcal{A}[\phi(u), \phi(v)]$ such that $u \in A_{i}$, each clique in $\mathcal{A}[\phi(u), i]$ also contains $u$.

By the construction of $\mathcal{S}_{u}$, this implies the following.

$\langle+\rangle$ each $A_{i} \in \mathcal{A}[\phi(u), \phi(v)]$ such that $u \in A_{i}$ satisfies $\lambda_{i} \in \mathcal{S}_{u}$.

Now, consider the path $P$ in $T$ from $A_{\phi(u)}$ to $A_{\phi(v)}$. Note that $u \in A_{\phi(u)}$ and $v \in A_{\phi(v)}$ by $(\star 0)$. Thus since $u v \in E(G)$ and since $T$ is a clique tree, there is some clique $Q$ on $P$ that contains both $u$ and $v$. We claim that there is an occurrence of $Q$ in $\mathcal{A}[\phi(u), \phi(v)]$. This is trivially true if $Q=A_{\phi(u)}$ or $Q=A_{\phi(v)}$. Otherwise, note that removing $Q$ from $T$ results in a forest in which $A_{\phi(u)}$ and $A_{\phi(v)}$ are in different components. Therefore, any walk in $T$ from $A_{\phi(u)}$ to $A_{\phi(v)}$ has to pass through $Q$. This implies that there is at least one occurrence of $Q$ in $\mathcal{A}[\phi(u), \phi(v)]$, which is a walk from $A_{\phi(u)}$ to $A_{\phi(v)}$ in $T$. Let $A_{s}$ be one of these occurrences.

Note that $u, v \in A_{s}=Q$. As $u \in A_{s}$ and since $A_{s} \in \mathcal{A}[\phi(u), \phi(v)]$, we conclude that $\lambda_{s} \in \mathcal{S}_{u}$ by $\langle+\rangle$. This implies that $\lambda_{s} \notin \mathcal{S}_{v}$, since $\mathcal{S}_{u} \cap \mathcal{S}_{v}=\varnothing$. Therefore, there exists some clique in $\mathcal{A}[s, \phi(v)]$ that does not contain $v$. Let $A_{j}$ be the last such clique in $\mathcal{A}[s, \phi(v)]$ and let $A_{i}$ be the last clique in $\mathcal{A}[s, j]$ that contains $v$. Recall that $v \in A_{\phi(v)}$ by $(\star 0)$. Thus the definition of $j$ implies that $v \in A_{j+1}$ and $v \in A_{i}$ but $v$ is in no clique in $\mathcal{A}(i, j+1)$. By $\langle 10\rangle$ (i), we now have $A_{i}=A_{j+1}$. Also by $\langle 10\rangle$ (iii), there exists $Q \in \mathcal{A}(i, j+1)$ that is a leaf of $T$. Using Lemma 8 , let $x$ be a vertex that is present in $Q$ and in no other clique in $V(T)$. Since $x$ appears in $A_{\phi(x)}$ by $(\star 0)$, this implies that $A_{\phi(x)}=Q$. Thus, we conclude that $A_{\phi(x)} \in \mathcal{A}(i, j+1)$, and hence, $A_{\phi(x)} \in \mathcal{A}(\phi(u), \phi(v))$, which yields $u x \in E(G)$ by $(\star 1)$. From this we deduce that $u \in Q$, since $Q$ is the only clique that contains $x$. Now, $\langle 10\rangle$ (ii) tells us that the path in $T$ between any clique in $\mathcal{A}[j+1, i]$ and any clique in $\mathcal{A}(i, j+1)$ passes through the clique $A_{i}=A_{j+1}$. As $u \in Q$ and $u \in A_{\phi(u)}$ by $(\star 0)$, the vertex $u$ is present in every clique on the path in $T$ between $A_{\phi(u)}$ and $Q$. This implies that $u \in A_{j+1}$. Hence, $\lambda_{j+1} \in \mathcal{S}_{u}$ by $\langle+\rangle$. For this recall that $A_{j+1} \in \mathcal{A}[\phi(u), \phi(v)]$. Now, since $A_{j}$ is the last clique in $\mathcal{A}[s, \phi(v)]$ that does not contain $v$, we also have $\lambda_{j+1} \in \mathcal{S}_{v}$ by the construction of $\mathcal{S}_{v}$. Thus we obtain $\mathcal{S}_{u} \cap \mathcal{S}_{v} \neq \varnothing$, which is a contradiction. This proves $\langle 13\rangle$.

This proves that $\left\{\mathcal{S}_{u}\right\}_{u \in V(G)}$ is indeed a circular-arc representation of $G$. Thus $G$ is a circular-arc graph as claimed. That concludes the proof.

As an illustration of the construction provided by this lemma, let us point the reader to Figure 4. In part a), we see a chordal graph, its clique tree, and an Euler tour of this clique tree (indicated by the labels $0,1, \ldots, 19)$. Part b) illustrates a choice of $\phi$ that satisfies the conditions of the lemma, and also shows the resulting circular-arc representation of the graph. Part c) shows a different mapping $\phi$ that fails the conditions and similarly shows corresponding circular arcs that fail to correctly represent the graph.

\subsection{Proof}

Finally, we are ready to give a proof of Theorem 4. Let $G$ be a chordal graph whose independence number $\alpha(G)$ is at most four. The direction (i) $\Rightarrow$ (ii) is proved as Lemma 1.

For the converse, assume (ii), that is, $G$ contains no blocking quadruple. In what follows, we describe how to conclude that (i) holds, namely that $G$ is a circular-arc graph. This splits into several cases.

First, if $\alpha(G) \leq 2$, then $G$ does not have an asteroidal triple. As $G$ is also chordal, $G$ is an interval graph [11], and hence, a circular-arc graph.

Next, suppose that $\alpha(G)=3$. As $G$ is a perfect graph, there is a clique cover $\mathcal{Q}=\left\{Q_{1}, Q_{2}, Q_{3}\right\}$ of $G$. Thus, by Lemma 9, any clique tree $T$ of $G$ can have at most 3 leaves. If $T$ has only 2 leaves, then $T$ is a path and therefore $G$ is an interval graph, and hence, a circular-arc graph. So, let us assume that $T$ has 3 leaves, which, in view of Lemma 9, are $Q_{1}, Q_{2}$ and $Q_{3}$. Let $\mathcal{A}=A_{0}, A_{1}, \ldots, A_{k-1}, A_{0}$ be any Euler tour of $T$. It is easy to see that each of $Q_{1}, Q_{2}, Q_{3}$, being a leaf of $T$, occurs exactly once in $\mathcal{A}$. For every vertex $u \in V(G)$, 

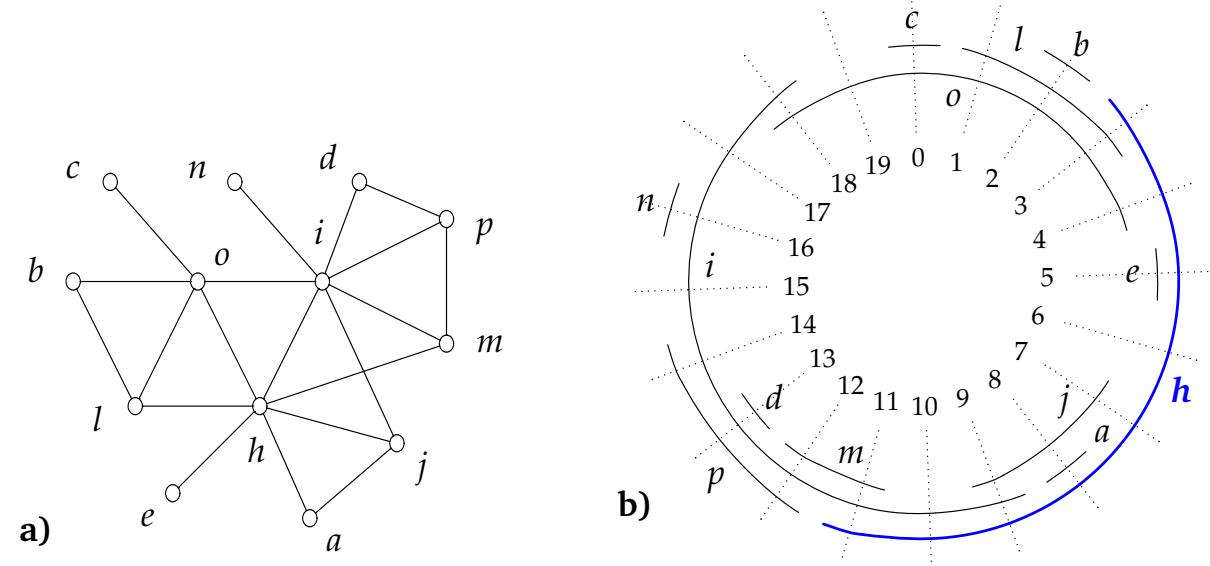

\begin{tabular}{c|ccc}
$v$ & $\phi(v)$ & $\ell_{v}$ & $r_{v}$ \\
\hline$a$ & 8 & 7 & 9 \\
$b$ & 2 & 1 & 3 \\
$c$ & 0 & 19 & 1 \\
$d$ & 13 & 12 & 14 \\
$e$ & 5 & 4 & 6 \\
$h$ & 6 & 2 & 12 \\
$i$ & 15 & 8 & 19 \\
$j$ & 8 & 6 & 10 \\
$l$ & 2 & 0 & 4 \\
$m$ & 12 & 10 & 13 \\
$n$ & 16 & 15 & 17 \\
$o$ & 1 & 17 & 5 \\
$p$ & 13 & 11 & 15
\end{tabular}
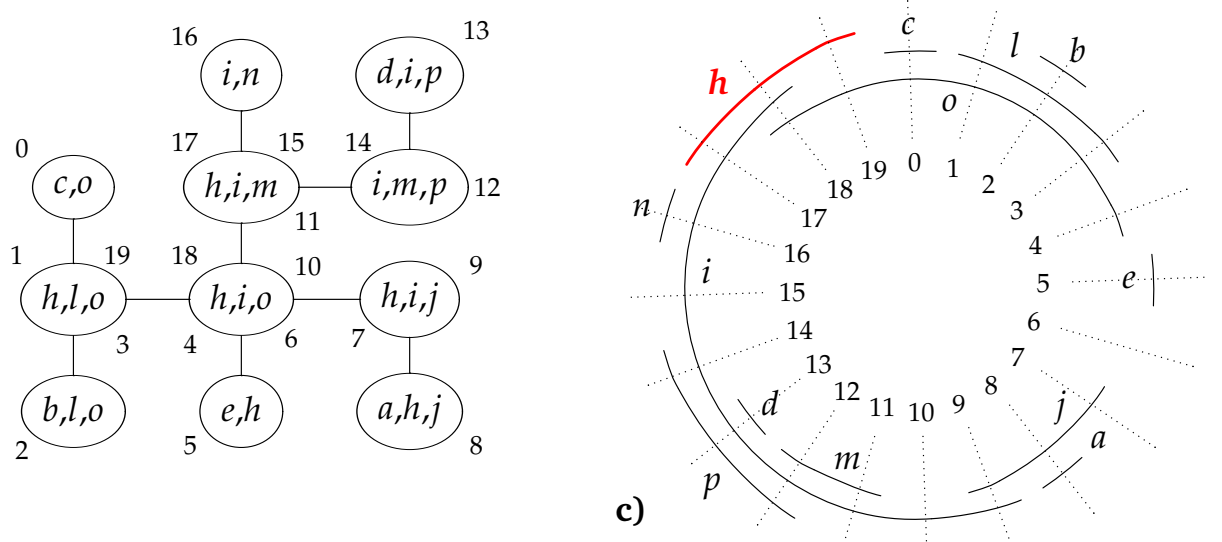

\begin{tabular}{c|ccc}
$v$ & $\phi(v)$ & $\ell_{v}$ & $r_{v}$ \\
\hline$a$ & 8 & 7 & 9 \\
$b$ & 2 & 1 & 3 \\
$c$ & 0 & 19 & 1 \\
$d$ & 13 & 12 & 14 \\
$e$ & 5 & 4 & 6 \\
$h$ & 18 & 16 & 0 \\
$i$ & 15 & 8 & 19 \\
$j$ & 8 & 6 & 10 \\
$l$ & 2 & 0 & 4 \\
$m$ & 12 & 10 & 13 \\
$n$ & 16 & 15 & 17 \\
$o$ & 1 & 17 & 5 \\
$p$ & 13 & 11 & 15
\end{tabular}

Figure 4: a) Example graph, b) correct choice of $\phi, c)$ incorrect choice

define $Q(u)$ to be any clique among $Q_{1}, Q_{2}, Q_{3}$ that contains $u$ (break ties arbitrarily). Then, define $\phi(u)$ to be the integer in $\{0,1, \ldots, k-1\}$ such that $A_{\phi(u)}$ is the unique occurrence of $Q(u)$ in $\mathcal{A}$.

Let us verify that the mapping $\phi$ satisfies the conditions of Lemma 10. Clearly, for each $u \in V(G)$, we have $u \in Q(u)$ by definition, and hence, $u \in A_{\phi(u)}$ since $A_{\phi(u)}=Q(u)$. Thus $\phi$ satisfies ( $\left.\star 0\right)$. Now, consider an edge $u v \in E(G)$. If $\phi(u)=\phi(v)$, then $\mathcal{A}(\phi(u), \phi(v))=\mathcal{A}(\phi(v), \phi(u))$ is empty, and so each of ( $\star 1)$ $(\star 4)$ is vacuously satisfied. So, assume $\phi(u) \neq \phi(v)$ in which case either $\mathcal{A}(\phi(u), \phi(v))$ or $\mathcal{A}(\phi(v), \phi(u))$ contains no clique of $\mathcal{Q}$. For this, note that $|\mathcal{Q}|=3$ and each clique in $\mathcal{Q}$ appears exactly once in $\mathcal{A}$, while $A_{\phi(u)}, A_{\phi(v)} \in \mathcal{Q}$ and $\phi(u) \neq \phi(v)$. Thus, since each $x \in V(G)$ satisfies $\mathcal{A}_{\phi(x)} \in \mathcal{Q}$ by the construction of $\phi$, we conclude that either $(\star 1)-(\star 2)$ or $(\star 3)-(\star 4)$ are vacuously satisfied. This verifies that $\phi$ satisfies the conditions of Lemma 10. Thus, by Lemma 10, we conclude that $G$ is a circular-arc graph.

We shall now prove the theorem for the case $\alpha(G)=4$. As before, since $G$ is a perfect graph, this means that $G$ can be covered with 4 cliques. Let $T$ be a clique tree of $G$ and let $\mathcal{Q}=\left\{Q_{1}, Q_{2}, Q_{3}, Q_{4}\right\}$ be a clique cover of $G$. By Lemma 9, the tree $T$ has at most 4 leaves. If $T$ has only 2 leaves, then $T$ is a path and therefore, $G$ is an interval graph (and hence a circular-arc graph).

Suppose that $T$ has 4 leaves. Then $Q_{1}, Q_{2}, Q_{3}, Q_{4}$ are the four leaves of $T$ and we have the two cases shown in Figure 5 a), b). For $i \in\{1,2,3,4\}$, since $Q_{i}$ is a leaf of the clique tree $T$, we know from Lemma 8 that there exists a vertex $v_{i} \in Q_{i}$ such that $Q_{i}$ is the only clique in $V(T)$ that contains $v_{i}$.

Now, consider the graph $H$ on $\left\{v_{1}, v_{2}, v_{3}, v_{4}\right\}$ with edge set

$$
E(H)=\left\{v_{i} v_{j} \mid v_{i}, v_{j} \text { avoid the vertices } V(H) \backslash\left\{v_{i}, v_{j}\right\}\right\}
$$




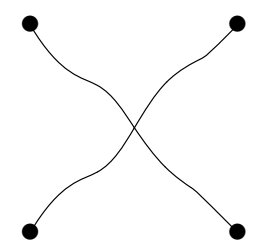

a)

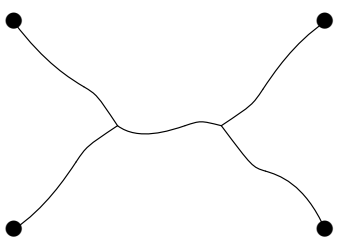

b)

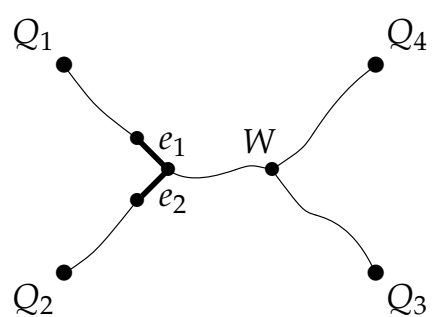

c)

Figure 5: When $T$ has 4 leaves

As $v_{1}, v_{2}, v_{3}, v_{4}$ is not a blocking quadruple, $H$ is either a 4-cycle, a $2 K_{2}$ or has no edges.

For an Euler tour $\mathcal{A}$ of $T$, we shall write $\left.\mathcal{A}\right|_{\mathcal{Q}}$ to denote the cyclic subsequence of $\mathcal{A}$ induced by the cliques in $\mathcal{Q}$. Namely, $\left.\mathcal{A}\right|_{\mathcal{Q}}$ is the cyclic permutation of $Q_{1}, Q_{2}, Q_{3}, Q_{4}$ that we obtain from $\mathcal{A}$ by removing all cliques but the occurrences of $Q_{1}, Q_{2}, Q_{3}, Q_{4}$.

Note that each $Q_{i}$, for $i \in\{1,2,3,4\}$, occurs exactly once in $\mathcal{A}$ as it is a leaf of $T$. We say that an Euler tour $\mathcal{A}$ respects $H$ if $Q_{i}$ and $Q_{j}$ are cyclically consecutive in $\left.\mathcal{A}\right|_{\mathcal{Q}}$ whenever $v_{i} v_{j} \in E(H)$. It turns out that the existence of such a tour implies that $G$ is a circular-arc graph. This is proved as follows.

$\langle *\rangle$ If some Euler tour of $T$ respects $H$, then $G$ is a circular-arc graph.

To see this, let $\mathcal{A}=A_{0}, A_{1}, \ldots, A_{k-1}, A_{0}$ be such a tour. Define $Q(u)$, for every $u \in V(G)$, to be any clique among $Q_{1}, Q_{2}, Q_{3}, Q_{4}$ that contains $u$ (break ties arbitrarily). Then, define $\phi(u)$ to be the integer in $\{0,1, \ldots, k-1\}$ such that $A_{\phi(u)}$ is the unique occurrence of $Q(u)$ in $\mathcal{A}$.

We claim that $\phi$ satisfies the conditions of Lemma 10. Clearly, for each $u \in V(G)$, we have $u \in \mathcal{A}_{\phi(u)}$, since $u \in Q(u)$ and $\mathcal{A}_{\phi(u)}=Q(u)$ by definition. Thus $\phi$ satisfies $(\star 0)$. Now, for contradiction, suppose that there exists an edge $u v \in E(G)$ that does not satisfy any of the conditions $(\star 1)-(\star 4)$.

Clearly, $Q(u) \neq Q(v)$ and $Q(u), Q(v)$ do not appear consecutively in $\left.\mathcal{A}\right|_{\mathcal{Q}}$, since otherwise either $\mathcal{A}(\phi(u), \phi(v))$ or $\mathcal{A}(\phi(v), \phi(u))$ contains no clique of $\mathcal{Q}$ and thus one of $(\star 1)-(\star 4)$ vacuously satisfied. So let us assume without loss of generality that $Q(u)=Q_{1}$ and $Q(v)=Q_{2}$. Then, $\left.\mathcal{A}\right|_{\mathcal{Q}}$ is the cyclic ordering $Q_{1}, Q_{3}, Q_{2}, Q_{4}$ or its reverse. As none of the conditions $(\star 1)-(\star 4)$ of Lemma 10 are satisfied for the edge $u v$, it follows that there exists a vertex $x_{3}$ with $Q\left(x_{3}\right)=Q_{3}$ and a vertex $x_{4}$ with $Q\left(x_{4}\right)=Q_{4}$ such that $u x_{3} \notin E(G)$ and $u x_{4} \notin E(G)$. From this, we conclude that $u \notin Q_{3}$ and $u \notin Q_{4}$. Similarly, there exists $y_{3}$ with $Q\left(y_{3}\right)=Q_{3}$ and $y_{4}$ with $Q\left(y_{4}\right)=Q_{4}$ such that $v y_{3}, v y_{4} \notin E(G)$. Thus $v \notin Q_{3}$ and $v \notin Q_{4}$. From this, since $Q_{3}$, resp. $Q_{4}$ is the only clique in $V(T)$ that contains $v_{3}$, resp. $v_{4}$, we conclude that $u v_{3}, v v_{3}, u v_{4}, v v_{4} \notin E(G)$. We now have a path $v_{1}, u, v, v_{2}$ that is missed by $v_{3}$ and by $v_{4}$ (note that it is possible that $u=v_{1}$ or $v=v_{2}$ ). Therefore, $v_{1}, v_{2}$ avoid $v_{3}, v_{4}$, which implies that $v_{1} v_{2} \in E(H)$. But $Q_{1}$ and $Q_{2}$ are not cyclically consecutive in $\left.\mathcal{A}\right|_{\mathcal{Q}}$, and so $\mathcal{A}$ does not respect $H$, a contradiction.

This shows that $\phi$ satisfies the conditions of Lemma 10. Thus by Lemma 10, we conclude that $G$ is a circular-arc graph. This proves $\langle *\rangle$.

In light of $\langle *\rangle$, it now suffices to show that an Euler tour of $T$ that respects $H$ exists. We analyze the two cases a) and b) of Figure 5 separately.

Let us first consider case a). Since $T$ consists of four paths joined at a single vertex, we have a freedom when traversing $T$ to choose to follow the four paths in any order. In other words, for every possible cyclic permutation of $Q_{1}, Q_{2}, Q_{3}, Q_{4}$, there is an Euler tour $\mathcal{A}$ of $T$ such that $\left.\mathcal{A}\right|_{\mathcal{Q}}$ is precisely the chosen permutation. From this, it follows that there exists an Euler tour $\mathcal{A}$ of $T$ that respects $H$. Namely, we choose a cyclic permutation of $Q_{1}, Q_{2}, Q_{3}, Q_{4}$ in which for non-consecutive cliques $Q_{i}, Q_{j}$ the pair $v_{i} v_{j}$ is not an edge of $H$. This is always possible as $H$ is isomorphic to a $C_{4}, 2 K_{2}$ or $4 K_{1}$. Therefore, by $\langle *\rangle$, we conclude that $G$ is a circular-arc graph.

If $G$ has a clique tree of the form a) from Figure 5, then we are done. Hence, we shall assume that $G$ has no clique tree of the form a). Suppose that $G$ has a clique tree of the form $b$ ) from Figure 5 . We claim 


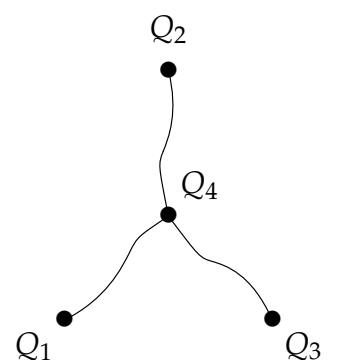

a)

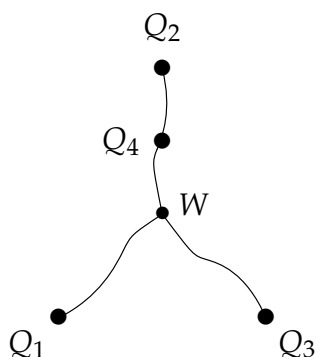

b)

Figure 6: When $T$ has only three leaves

that there is an Euler tour $\mathcal{A}$ of $T$ that respects $H$. Let the leaves of the tree $T$ be labelled as shown in $\mathrm{c}$ ) in Figure 5 and let $W$ be the vertex of $T$ as shown in the figure.

It follows that there is no Euler tour of $T$ that respects $H$ if and only if $H$ is the cycle $v_{3}, v_{1}, v_{4}, v_{2}$. Suppose that this is the case. Note that $v_{1} v_{2} \notin E(H)$. Let $e_{1}$ and $e_{2}$ be the edges shown in c) in Figure 5 , let $C$ be their common endpoint, and $C_{1}$ resp. $C_{2}$ be their other endpoints, i.e., $e_{1}=C C_{1}$ and $e_{2}=C C_{2}$. We claim that either $C \cap C_{1} \subseteq W$ or $C \cap C_{2} \subseteq W$. Indeed, since $T$ is a clique tree, we know that there exists a vertex $x_{1} \in C_{1} \backslash C$ and a vertex $x_{2} \in C_{2} \backslash C$. It follows that $x_{1} \in Q_{1}$ and $x_{2} \in Q_{2}$, since $T$ is a clique tree, $x_{1}, x_{2} \notin C$, and since $\mathcal{Q}$ is a clique cover of $G$. Now, if there exist vertices $u_{1} \in\left(C \cap C_{1}\right) \backslash W$ and $u_{2} \in\left(C \cap C_{2}\right) \backslash W$, then $v_{1}, x_{1}, u_{1}, u_{2}, x_{2}, v_{2}$ is a path in $G$ from $v_{1}$ to $v_{2}$ that is missed by both $v_{3}$ and $v_{4}$. But then $v_{1}, v_{2}$ avoid $v_{3}, v_{4}$ implying $v_{1} v_{2} \in E(G)$, a contradiction. Thus, from $C \cap C_{1} \subseteq W$ or $C \cap C_{2} \subseteq W$, we conclude that either the tree obtained from $T$ by removing $e_{1}$ and adding the edge $C_{1} W$ or the tree obtained from $T$ by removing $e_{2}$ and adding the edge $C_{2} W$ is another clique tree of $G$. However, both these trees are of the form a), and we assume that $G$ has no such clique tree, a contradiction.

We can therefore conclude that there is an Euler tour of $T$ that respects $H$. Thus, by $\langle *\rangle$, we again conclude that $G$ is a circular-arc graph.

If $G$ has some clique tree that has four leaves, we are done. Thus, we shall assume that every clique tree of $G$ has exactly 3 leaves. Let $T$ be any clique tree of $G$. In this case, $T$ is of one of the two forms in Figure 6.

In both these cases, one of the cliques in $\mathcal{Q}=\left\{Q_{1}, Q_{2}, Q_{3}, Q_{4}\right\}$ is an internal node of $T$. Let us assume without loss of generality that $Q_{4}$ is the internal node among these in both cases. For $i \in\{1,2,3\}$, we let $v_{i}$ be a vertex that belongs to the clique $Q_{i}$ and to no other clique in $V(T)$. We let $v_{4}$ be a vertex in $Q_{4}$ that does not belong to any other clique of $\mathcal{Q}$. Its existence is guaranteed by the fact that $G$ has no clique cover of size 3 (as $\alpha(G)=4$ ). For every vertex $u \in Q_{1} \cup Q_{2} \cup Q_{3}$, we define $Q(u)$ so that $u \in Q(u)$. For a vertex $u \in V(G)$, we set $Q(u)=Q_{4}$ if and only if $u \in Q_{4} \backslash\left(Q_{1} \cup Q_{2} \cup Q_{3}\right)$.

Let $\mathcal{A}=A_{0}, A_{1}, \ldots, A_{k-1}, A_{0}$ be an Euler Tour of $T$. We again seek to construct $\phi$. For vertices $u$ with $Q(u) \in\left\{Q_{1}, Q_{2}, Q_{3}\right\}$, the clique $Q(u)$ is a leaf of $T$ and thus appears exactly once in $\mathcal{A}$. We choose this occurrence for $A_{\phi(u)}$ in order to satisfy $(\star 0)$. For vertices $u$ with $Q(u)=Q_{4}$, we have more freedom as $Q_{4}$ appears multiple times in any Euler tour of $T$. Namely, it appears exactly three times if $T$ is of the form a) of Figure 6, and it appears exacty two times if $T$ is of the form b). We choose one of these occurrences and assign $\phi(u)$ so that $A_{\phi(u)}$ is this occurrence for every $u$ with $Q(u)=Q_{4}$. The choice of this occurrence, however, will not be arbitrary. We will again use the graph $H$ as constructed in the case with four leaves in $T$.

Namely, let $H$ be the graph on vertices $\left\{v_{1}, v_{2}, v_{3}, v_{4}\right\}$ where $v_{i} v_{j}$ is an edge if and only if $v_{i}, v_{j}$ avoid $V(H) \backslash\left\{v_{i}, v_{j}\right\}$. Since $v_{1}, v_{2}, v_{3}, v_{4}$ is not a blocking quadruple, we conclude that $H$ is either a 4-cycle, a $2 K_{2}$, or edgeless. Also, as in the case of four leaves, write $\left.\mathcal{A}\right|_{\mathcal{Q}}$ to denote the cyclical sequence of the elements of $\mathcal{Q}$ that is obtained from $\mathcal{A}$ by removing all occurrences of cliques that are not in $\mathcal{Q}$.

First, suppose that $T$ is of the form a). Since $H$ contains no triangle, one of the pairs $v_{1} v_{2}, v_{2} v_{3}, v_{1} v_{3}$ is not an edge of $H$. By symmetry, without loss of generality, we may assume that $v_{1} v_{2} \notin E(H)$. Also, without loss of generality, we may assume that $\mathcal{A}$ is an Euler tour of $T$ such that

$$
\left.\mathcal{A}\right|_{\mathcal{Q}}=Q_{1}, Q_{4}, Q_{2}, Q_{4}, Q_{3}, Q_{4}
$$


(If not, we simply reverse and/or cyclically shift $\mathcal{A}$ to achieve this.)

For every vertex $u \in V(G)$ such that $Q(u)=Q_{4}$, define $\phi(u)$ to be the integer such that $A_{\phi(u)}$ is the occurrence of $Q_{4}$ in $\mathcal{A}$ after $Q_{1}$ and before $Q_{2}$. For every vertex $u \in V(G)$ such that $Q(u)=Q_{i}$, where $i \in\{1,2,3\}$, define $\phi(u)$ to be the integer such that $A_{\phi(u)}$ is the unique occurrence of $Q_{i}$ in $\mathcal{A}$.

Let us now verify that $\phi$ satisfies the conditions of Lemma 10. By definition, $u \in A_{\phi(u)}$ for all $u \in V(G)$. Thus $\phi$ satisfies $(\star 0)$. Now, consider an edge $u v \in E(G)$. If $Q(u)=Q(v)$ or if $Q(u) \in\left\{Q_{1}, Q_{2}\right\}$ and $Q(v) \in\left\{Q_{3}, Q_{4}\right\}$, then either $\mathcal{A}(\phi(u), \phi(v))$ or $\mathcal{A}(\phi(v), \phi(u))$ contains only an occurrence $A_{i}$ of $Q_{4}$ (if any) among the cliques of $\mathcal{Q}$, but no $x \in V(G)$ satisfies $\phi(x)=i$ by the definition of $\phi$. Thus one of $(\star 1)-(\star 4)$ is vacuously satisfied in this case. The same holds by symmetry if $Q(u) \in\left\{Q_{3}, Q_{4}\right\}$ and $Q(v) \in\left\{Q_{1}, Q_{2}\right\}$. Thus we may assume that $Q(u) \neq Q(v)$ and either $Q(u), Q(v) \in\left\{Q_{1}, Q_{2}\right\}$ or $Q(u), Q(v) \in\left\{Q_{3}, Q_{4}\right\}$.

Suppose first that $Q(u), Q(v) \in\left\{Q_{3}, Q_{4}\right\}$. By symmetry, we may assume that $Q(u)=Q_{3}$ and $Q(v)=$ $Q_{4}$. If $u v$ fails all of $(\star 1)$ - $(\star 4)$, then there exist vertices $x_{1}, x_{2}, y_{1}, y_{2}$ where $Q\left(x_{1}\right)=Q\left(y_{1}\right)=Q_{1}$ and $Q\left(x_{2}\right)=Q\left(y_{2}\right)=Q_{2}$ such that $u x_{1}, u x_{2}, v y_{1}, v y_{2} \notin E(G)$. From this, we deduce $u, v \notin Q_{1}$ and $u, v \notin Q_{2}$. Therefore $u v_{1}, u v_{2}, v v_{1}, v v_{2} \notin E(G)$, since $Q_{1}$, resp. $Q_{2}$ is the only clique that contains $v_{1}$, resp. $v_{2}$. Thus $v_{3}, u, v, v_{4}$ is a path (possibly $u=v_{3}$ or $v=v_{4}$ ) missed by $v_{1}$ and $v_{2}$. This yields $v_{1} v_{2} \in E(H)$, a contradiction.

Next, suppose that $Q(u), Q(v) \in\left\{Q_{1}, Q_{2}\right\}$. By symmetry, assume that $Q(u)=Q_{1}$ and $Q(v)=Q_{2}$. If $u v$ fails $(\star 1)-(\star 4)$, then there are vertices $x_{4}, y_{4}$ with $Q\left(x_{4}\right)=Q\left(y_{4}\right)=Q_{4}$ such that $u x_{4}, v y_{4} \notin E(G)$. This implies $u, v \notin Q_{4}$. Let $P$ be the path of $T$ between $Q_{1}$ and $Q_{2}$. Since $T$ is as depicted in Figure 6 , the clique $Q_{4}$ lies on $P$. Also, since $T$ is a clique tree and $u v \in E(G)$ where $u \in Q_{1}$ while $v \in Q_{2}$, there exists a clique $Q$ on $P$ such that $u, v \in Q$. Thus either $u \in Q_{4}$ if $Q_{4}$ belongs to the subpath of $P$ between $Q_{1}$ and $Q$, or $v \in Q_{4}$ if otherwise. However, earlier we deduced that $u, v \notin Q_{4}$, a contradiction.

This shows that $\phi$ satisfies the conditions of Lemma 10. Thus by Lemma 10, $G$ is a circular-arc graph.

Finally, suppose that $T$ is of the form b) of Figure 6. Let the cliques $Q_{1}, Q_{2}, Q_{3}, Q_{4}$ be as labelled in the figure. Let $P$ be the path between $Q_{4}$ and $Q_{2}$ in T. Let $Q_{2}^{\prime}$ be the neighbour of $Q_{4}$ on $P$ (possibly $Q_{2}^{\prime}=Q_{2}$ ).

We prove that the vertices $v_{1}, v_{3}$ avoid the vertices $v_{2}, v_{4}$ in $G$. Firstly, suppose there exists a vertex $u \in Q_{2}^{\prime}$ with $Q(u)=Q_{4}$. Since $Q_{4}$ and $Q_{2}^{\prime}$ are distinct maximal cliques of $G$, there exists $u^{\prime} \in Q_{2}^{\prime} \backslash Q_{4}$. We claim that $Q\left(u^{\prime}\right)=Q_{2}$ and $u^{\prime} \notin Q_{1} \cup Q_{3}$. Indeed, if $u^{\prime}$ belongs to $Q_{1}$ or $Q_{3}$, then it also belongs to $Q_{4}$, since $T$ is a clique tree, and the path between $Q_{1}, Q_{3}$ and $Q_{2}^{\prime}$ goes through $Q_{4}$. But $u^{\prime} \notin Q_{4}$. So we must conclude $u^{\prime} \notin Q_{1} \cup Q_{3}$, and hence, $Q\left(u^{\prime}\right)=Q_{2}$ because $Q_{2}$ is the only remaining clique from $\mathcal{Q}$ that can contain $u^{\prime}$. We also have $u \notin Q_{1} \cup Q_{3}$, since $Q(u)=Q_{4}$ if and only if $u \in Q_{4} \backslash\left(Q_{1} \cup Q_{2} \cup Q_{3}\right)$, by definition. From this, we deduce that $u v_{1}, u^{\prime} v_{1}, u v_{3}, u^{\prime} v_{3} \notin E(G)$, since $Q_{1}$, resp. $Q_{3}$ is the only clique in $V(T)$ that contains $v_{1}$, resp. $v_{3}$. This implies that $v_{4}, u, u^{\prime}, v_{2}$ forms a path in $G$ that is missed by both $v_{1}$ and $v_{3}$ (note that possibly $u^{\prime}=v_{2}$ ). Thus we conclude that $v_{1}, v_{3}$ avoid $v_{2}, v_{4}$ as promised.

Secondly, suppose there exists a vertex $u \in Q_{4} \cap Q_{2}$ that does not belong to $Q_{1} \cup Q_{3}$. Then $v_{4}, u, v_{2}$ forms a path in $G$ that is missed by both $v_{1}$ and $v_{3}$. Thus, we again conclude that $v_{1}, v_{3}$ avoid $v_{2}, v_{3}$.

We claim that one of these cases must be fulfilled. Indeed, suppose that every $u \in Q_{2}^{\prime}$ satisfies $Q(u) \in$ $\left\{Q_{1}, Q_{2}, Q_{3}\right\}$, and every $u \in Q_{4} \cap Q_{2}$ belongs to $Q_{1} \cup Q_{3}$. Thus every $u \in Q_{4} \cap Q_{2}^{\prime}$ is in $Q_{1} \cup Q_{3}$. Namely, if $Q(u) \in\left\{Q_{1}, Q_{3}\right\}$, then $u \in Q_{1} \cup Q_{3}$ by definition, and if $Q(u)=Q_{2}$, then $u \in Q_{2}$, and hence, $u \in Q_{4} \cap Q_{2}$ implying $u \in Q_{1} \cup Q_{3}$ by our assumption. Since every such $u$ is in $Q_{1} \cup Q_{3}$, it also belongs to $W$, since $T$ is a clique tree and $W$ lies on the path of $T$ between $Q_{4}$ and either of $Q_{1}, Q_{3}$. This means that the tree obtained by removing the edge $Q_{4} Q_{2}^{\prime}$ from $T$ and adding the edge $W Q_{2}^{\prime}$ is again a clique tree of $G$. But this is a clique tree of $G$ with four leaves, and we assume that $G$ has no such clique tree, a contradiction.

Now, since $v_{1}, v_{3}$ avoid $v_{2}, v_{4}$, we have that $v_{1} v_{3}$ and $v_{2} v_{4}$ are edges of $H$. Since $H$ contains no triangle, this means that one of $v_{1} v_{2}, v_{2} v_{3}$ is not an edge of $H$. By symmetry, without loss of generality, assume that $v_{1} v_{2} \notin E(H)$. Also, without loss of generality, assume that $\mathcal{A}$ is an Euler tour of $T$ such that

$$
\left.\mathcal{A}\right|_{\mathcal{Q}}=Q_{1}, Q_{4}, Q_{2}, Q_{4}, Q_{3}
$$

For every vertex $u \in V(G)$ such that $Q(u)=Q_{4}$, define $\phi(u)$ to be the integer such that $A_{\phi(u)}$ is the occurrence of $Q_{4}$ in $\mathcal{A}$ after $Q_{1}$ and before $Q_{2}$. For every vertex $u \in V(G)$ such that $Q(u)=Q_{i}$, where $i \in\{1,2,3\}$, define $\phi(u)$ to be the integer such that $A_{\phi(u)}$ is the unique occurrence of $Q_{i}$ in $\mathcal{A}$. 
Notice that this definition of $\phi$ is identical to that of the previous case when $T$ was assumed to be type a). We also assume that $v_{1} v_{2} \notin E(H)$. This allows us to repeat the argument (word-for-word) from the previous case that shows that $\phi$ satisfies the conditions of Lemma 10. From this, we conclude by Lemma 10 that $G$ is a circular-arc graph. This exhausts all cases and thus concludes the proof of Theorem 4.

\section{References}

[1] BAng-Jensen, J., AND Hell, P. On the chordal proper circular-arc graphs. Discrete Mathematics 128 (1994), $395-398$.

[2] Bonomo, F., Durán, G., GRIPpo, L. N., AND SAFE, M. D. Partial characterizations of circular-arc graphs. Journal of Graph Theory 61 (2009), 289-306.

[3] Booth, K. S., AND LUEKeR, G. S. Testing for the consecutive ones property, interval graphs and graph planarity using PQ-tree algorithms. Journal of Computer System Sciences 13 (1976), 335-379.

[4] BRAndstÄDT, A., AND HoÀnG, C. T. On clique separators, nearly chordal graphs, and the maximum weight stable set problem. Theoretical Computer Science 389 (2007), 295-306.

[5] Corneil, D. G., Olariu, S., And StewART, L. The LBFS structure and recognition of interval graphs. SIAM Journal on Discrete Mathematics 23 (2009), 1905-1953.

[6] Feder, T., Hell, P., ANd HuAng, J. List homomorphisms and circular-arc graphs. Combinatorica 19 (1999), 487-505.

[7] GAVRIL, F. The intersection graphs of subtrees in trees are exactly the chordal graphs. Journal of Combinatorial Theory $B 16$ (1974), 47-56.

[8] Gilmore, P. C., AND Hoffman, A. J. A characterization of comparability graphs and of interval graphs. Canadian Journal of Mathematics 16 (1964), 539-548.

[9] KLEE, V. What are the intersection graphs of arcs in a circle ? American Mathematical Monthly 76 (1976), 810-813.

[10] Kratsch, D., McConnell, R., Mehlhorn, K., And Spinrad, J. Certifying algorithms for recognizing interval graphs and permutation graphs. SIAM Journal on Computing 36 (2006), 326-353.

[11] LeKkerkerker, C. G., AND Boland, J. C. Representation of a finite graph by a set of intervals on the real line. Fundamenta Mathematicae 51 (1962), 45-64.

[12] Lin, M. C., AND SzWARCFITER, J. L. Characterizations and linear time recognition of Helly circular-arc graphs. In Proceedings of the 12th Annual International Conference on Computing and Combinatorics (COCOON 2006), Lecture Notes in Computer Science 4112 (2006), pp. 73-82.

[13] Trotter, W. T., AND Moore, J. I. Characterizations problems for graphs, partially ordered sets, lattices and families of sets. Discrete Mathematics 16 (1976), 361-381.

[14] Tucker, A. Two characterizations of proper circular-arc graphs. PhD thesis, Stanford University, Stanford, California, USA, 1969.

[15] Tucker, A. Characterizing circular-arc graphs. Bulletin of the American Mathematical Society 76 (1970), 1257-1260.

[16] Tucker, A. Structure theorems for some circular-arc graphs. Discrete Mathematics 7 (1974), $167-195$. 